\title{
NECESIDADES DE ORIENTACIÓN EN LA FORMACIÓN INICIAL DE ESTUDIANTES DE LA ESCUELA DE FORMACIÓN DOCENTE DE LA UNIVERSIDAD DE COSTA RICA
}

\author{
Ileana Blanco Solís \\ Yorleny Jara Vázquez \\ Jorge Navarro Alfaro* \\ Aida Rosa Segura Calvo*
}

\begin{abstract}
Resumen: La formación inicial del grupo de profesionales en educación, exige hoy más que nunca de servicios efectivos de Orientación en la comunidad universitaria, puesto que los cambios económicos, las transformaciones sociales, las demandas del mercado de trabajo y los requerimientos de las profesiones, plantean un futuro difícil para la población estudiantil universitaria. Ante esta realidad, se realizó una investigación para dar respuesta al siguiente problema.

De acuerdo con las percepciones de un grupo de estudiantes de la Escuela de Formación Docente de la Universidad de Costa Rica, ¿qué necesidades de orientación se encuentran asociadas a su formación inicial para enfrentar constructivamente los cambios, demandas y desafíos del Sistema Educativo Costarricense? El paradigma de investigación utilizado comprende la investigación social cualitativa. Para aplicar esta metodología se utilizó como técnica de recolección de la información, los grupos de discusión y el análisis de contingencias como técnica de análisis de la información. El logro de los objetivos de la investigación permitió identificar las siguientes necesidades de orientación en la población estudiada: autoafirmación profesional, habilidades de vida y madurez vocacional.
\end{abstract}

Palabras clave: EDUCACIÓN SUPERIOR/ ORIENTACIÓN EDUCATIVA/ NECESIDADES DE ORIENTACIÓN/ FORMACIÓN INICIAL DE EDUCADORES/

\begin{abstract}
The initial training undergone by the group of professionals in the field of education demands today more than ever effective counseling services within the university community, due to the fact that changes in the economy, social transformations, the demands of the labor market and requirements for professionals create a difficult future for the university student population.

The investigation tried to answer the following question in accordance to the viewpoints of a group of students from the Escuela de Formación Docente of the University of Costa Rica, Which counseling needs are linked to your initial training in order to constructively face the changes, demands and challenges of the educational system of Costa Rica? The investigation paradigm that was used was the Qualitative Social Investigation. In order to apply this methodology, discussion groups were used as a means to gather information and the analysis of contingencies was used as an analysis technique. The accomplishment of the objectives of the investigation made it possible to identify the following needs for Counseling in the studied population: professional self affirmation, life skills and vocational maturity.
\end{abstract}

Key words: HIGHER EDUCATION/ SCHOOL COUNSELING/ DEVELOPMENTAL NEEDS/ PRE SERVICE EDUCATION/

\footnotetext{
* Licenciatura en Ciencias de la Educación con énfasis en Orientación, de la Universidad de Costa Rica. Profesionales en ejercicio en instituciones de educación pública.
}

Correo electrónico de referencia: amatas@cariari.ucr.ac.cr

Artículo recibido: 19 de octubre, 2005 Aprobado: 12 de diciembre, 2005 


\section{INTRODUCCION}

De acuerdo con Archer y Cooper (1998), la necesidad de servicios efectivos de orientación para la comunidad estudiantil universitaria nunca ha sido mayor que ahora. Los cambios económicos y sociales, la naturaleza cambiante del mercado de trabajo y las transiciones que enfrenta la sociedad ante un mundo globalizado, plantean un futuro difícil para la juventud profesional. Complementan esta situación la trasformación de las estructuras familiares y el aumento en la visibilidad de los problemas sociales como la pobreza, la violencia, el racismo, el abuso del alcohol y las drogas, lo que determina en gran medida la calidad de los años de formación del estudiantado universitario.

La sociedad costarricense no escapa de esta realidad. De acuerdo con el Octavo Informe del Estado de la Nación en Desarrollo Humano Sostenible (Estado de la Nación, 2002), desde hace varios años la distribución del ingreso, los niveles de pobreza, subempleo y desempleo en el país, vienen mostrando pequeños deterioros anuales que se han ido acumulando y que han impactado negativamente la cobertura de los servicios de salud y educación, la provisión de servicios básicos, y la equidad social. De manera contradictoria, este mismo informe destaca un leve mejoramiento macroeconómico, y como aspecto relevante para sustentar este trabajo, el surgimiento de nuevos grupos organizados de la sociedad civil que buscan hacer efectiva su participación social, mediante mecanismos recién implementados en la dinámica sociopolítica del país.

De este panorama sugiere que la participación social efectiva requeriría de un nivel superior de escolaridad de la población, mediante la formación profesional, objeto del quehacer universitario. Pero no es suficiente este incremento para el logro de una participación social efectiva. La educación superior debe procurar una formación renovada e innovadora que fortalezca la capacidad de análisis y de diálogo; la promoción del liderazgo en los futuros grupos de profesionales; el fortalecimiento de su desarrollo personal y académico con miras a la construcción de personalidades saludables y por lo tanto, una ciudadanía productiva; el compromiso con el desarrollo teórico de la profesión; y el enfrentamiento responsable y constructivo de dilemas éticos y morales.

Dentro de este contexto y de manera específica, al grupo de profesionales en educación se les exige hoy una actuación más pertinente; esta demanda se sustenta en el aparente o real deterioro del sistema educativo, lo que ocasiona en este grupo profesional un sentimiento de inadecuación para la tarea docente. Esta situación no sólo es producto del modelo aplicado en el proceso de formación inicial del profesorado, sino que recientemente las exigencias a este sector profesional, se van incrementando debido a las trasformaciones 
como consecuencia del cambio social, y que exigen a este grupo de profesionales de la educación una adecuación de sus tareas. De acuerdo con Esteve, Franco y Vera (1995), en los últimos 20 años, en el ámbito europeo, diversos cambios sociales, políticos y económicos han configurado un panorama muy distinto para el profesorado. Los autores describen esta experiencia utilizando la imagen de un grupo de actores de una obra clásica, a quienes se les cambia radicalmente el decorado del escenario para representar un ambiente posmodernista. No obstante continúan vestidos a la usanza de épocas anteriores y en pleno desarrollo de su obra teatral sin lograr hacer ninguna transformación a su diálogo.

Una situación similar, en términos de impacto del cambio, enfrentan el profesorado costarricense. De acuerdo con el Octavo Informe del Estado de la Nación en Desarrollo Humano Sostenible (Estado de la Nación, 2002), las condiciones económicas y sociales del país se polarizan cada vez más y a mayor velocidad, la inversión en educación se ha incrementado pero en términos nominales, las expectativas acerca de la democracia costarricense incluyen con mayor amplitud el acceso a educación de buena calidad, se deteriora el papel de la familia en medio de influencias externas de gran empuje, entre otras condiciones. De este informe se pueden interpretar los elementos de cambio a los que se enfrentan el grupo de profesionales en educación, concretándose en algunos de ellos: la escasez de recursos económicos para la inversión en infraestructura escolar; el incremento en la tasa de embarazo adolescente; el incremento del número de estudiantes con condiciones asociadas a discapacidad; el aumento significativo en los últimos años de los inmigrantes nicaragüenses que exigen al sistema educativo una actuación más pertinente a sus necesidades; y los constantes niveles de deserción escolar.

Ante esta realidad se realizó una investigación que busca de dar respuesta al siguiente problema: De acuerdo con las percepciones de un grupo de estudiantes de la Escuela de Formación Docente de la Universidad de Costa Rica, ¿qué necesidades de orientación se encuentran asociadas a su formación inicial para enfrentar constructivamente los cambios, demandas y desafíos del Sistema Educativo Costarricense?

\section{Como objetivos de la investigación se plantearon los siguientes:}

- Identificar las necesidades de orientación de un grupo de estudiantes de la Escuela de Formación Docente de la Universidad de Costa Rica de acuerdo con su propia perspectiva y los cambios sociales que se afrontan en el Sistema Educativo Costarricense. 
- Reconocer las formas o estrategias que mayormente responderían a las necesidades mostradas por los sujetos de estudio.

El presente trabajo constituye un aporte que permitirá la actualización universitaria en dos grandes vías. La primera se refiere a la identificación de manera específica de las necesidades de orientación del estudiantado de las carreras de Formación Docente de la Facultad de Educación de la Universidad de Costa Rica. Estas necesidades se encuentran asociadas a las condiciones psicosociales de este grupo de estudiantes, en función a su formación inicial y su perspectiva sobre el futuro desempeño profesional. Como segunda vía, la construcción de este objeto de estudio permitió reconocer las formas o estrategias de Orientación que mayormente responderían a las necesidades mostradas por la población estudiantil participante del estudio.

En síntesis, la investigación buscó tener información para facilitar la atención particular de las necesidades de orientación de la población estudiantil de las carreras de Formación Docente en su período de formación inicial, por parte de la Oficina de Orientación y del profesorado universitario que atiende a esta población.

\section{ASPECTOS TEÓRICOS}

El sustento teórico del trabajo abarca tres aspectos que facilitan la comprensión y el análisis de los datos recopilados. El primer tema está referido a la formación inicial profesional docente con el propósito de comprender la trayectoria de la formación docente a la luz de los cambios actuales, y la respuesta curricular frente a estos cambios. El segundo tema comprende el desarrollo vocacional del grupo de profesionales en educación, en vista de que, al ser un estudio que busca encontrar necesidades de orientación, el enfoque de desarrollo humano es el que sustenta coherentemente esta perspectiva de la orientación. Más adelante se analiza el papel de la Orientación en la universidad como respuesta a las necesidades de orientación del estudiantado, y los agentes facilitadores de la orientación, con el propósito de identificar los aportes que estos brindan a la formación de la población estudiantil, y así hacer un análisis integral y contextualizado a la realidad del estudiantado universitario. De manera muy breve se expondrán algunos elementos de los temas mencionados.

\section{Formación inicial del profesorado}


Apodaca y Lobato (1997) hacen referencia a que la calidad educativa se mide por la diferencia en el grado de desarrollo intelectual, afectivo, personal y social del profesorado antes y después de su formación inicial. Por lo tanto la población estudiantil universitaria debe alcanzar: comprensión y aprecio por las diferencias humanas; competencia en la resolución de conflictos; un coherente e integrado sentido de identidad, autoestima, confianza, integridad, sensibilidad, estética, y responsabilidad como ciudadano o ciudadana; desarrollo de actitudes, valores, perspectivas y capacidades para un continuo aprendizaje; incremento de la madurez emocional, tolerancia, empatía y habilidades de liderazgo; complejas habilidades cognitivas como la reflexión y el pensamiento crítico; habilidades para aplicar los conocimientos en problemas prácticos del cambio profesional, familiar, personal y social; y criticidad para evaluar lo aprendido.

El desarrollo profesional del profesorado se define como un proceso amplio y flexible, caracterizado por diferentes etapas evolutivas. Y a su vez, la formación de este grupo de profesionales es un proceso continuo, sistemático y organizado, que abarca toda la carrera docente (Marcelo, 1994). Feiner (Marcelo, 1994) menciona como etapas de desarrollo profesional las siguientes. La primera se denomina fase de preentrenamiento constituida por las enseñanzas previas que se han vivido como alumnos, de forma acrítica y que influyen inconscientemente. Una segunda es denominada fase de formación inicial que se desarrolla cuando la persona se prepara formalmente en una institución específica, adquiriendo conocimientos pedagógicos, de disciplinas académicas y prácticas. Una tercera se denomina fase de iniciación que representa el primer año de ejercicio profesional, donde se aprende en la práctica por estrategias de supervivencia. Finalmente, se da la fase permanente en la que todas las actividades están planificadas por las instituciones para fomentar el desarrollo profesional y perfeccionamiento, es decir, la actualización profesional.

Para efectos de la investigación, se enfatiza en la formación inicial, la cual, según Imbernón (1994), se caracteriza por ser una etapa de cambios y trasformaciones, que debe dotar al estudiantado en docencia de destrezas y actitudes que le permitan alterar, recrear y reconvertir el contexto educativo. En consecuencia, la formación inicial debe dotar al estudiantado de un sólido bagaje cultural, psicopedagógico y personal. Sin embargo, dentro de las situaciones problemáticas que se presentan en la formación del profesorado se pueden mencionar la inexistencia de un único modelo de formación, el reto que significa formarles tanto en cuanto a la aplicación de técnicas pedagógicas específicas como en principios pedagógicos, la influencia de las experiencias educativas previas con docentes antes de la fase formativa, la necesidad de considerar la diversidad de poblaciones que 
acceden a la formación universitaria lo que genera un sinnúmero de necesidades estudiantiles, y el poco control sobre el perfil del profesorado universitario.

La formación inicial va a pernear, en gran manera, los primeros años laborales del profesorado principiante. Pasar de la universidad a la práctica profesional conlleva un choque con la realidad que se caracteriza por la confrontación de la imagen elaborada en los años de formación y la adopción de las situaciones reales en el aula. Esto implica una autoconfrontación con su desarrollo profesional y un paso adelante en la socialización profesional que contribuye a moldear la imagen de la profesión que acabará orientando las actitudes, hábitos y expectativas del profesorado (Esteve, Franco y Vera, 1995).

Es importante hacer referencia a la situación actual del profesorado tomando como base el proceso histórico y cultural en el cual se desenvuelven, debido a que los cambios sociales transforman el trabajo que este grupo de profesionales realiza, su imagen social y la valoración que la sociedad hace de la educación. Esteve, Franco y Vera (1995) enumeran doce indicadores básicos en los que resumen los principales cambios de la educación en los últimos veinte años en Europa y la influencia de estos factores en la práctica docente actual. A continuación, se hace referencia a algunos de estos indicadores y su posible relación con la realidad costarricense.

- Expectativas de la sociedad y aumento de exigencias sobre el profesorado, al tener que asumir un mayor numero de responsabilidades que lleven a perfeccionar la personalidad del estudiantado, tales como atender las necesidades educativas especiales.

- Inhibición educativa de otros agentes de socialización, como el debilitamiento del rol de la familia y otras instituciones de sociales y comunitarias, lo cual le exige a los profesionales y las profesionales en educación, tareas que deberían ser compartidas, como la formación de hábitos y valores.

- Modificación del apoyo de la sociedad al sistema educativo; en los últimos veinte años y, en buena medida por la ruptura del consenso sobre los fines y principios de la educación, han aumentado las contradicciones con respecto al ejercicio de la función docente.

- Descenso en la valoración social del grupo de profesionales en educación, al valorarse en la sociedad actual el estatus social con un mayor nivel de ingresos que a los ideales de vocación y abnegación propios de la labor docente.

- Cambio en los contenidos curriculares; que exigen trasformaciones actitudinales y conceptuales, sin una previa preparación, creando inseguridad en el profesorado. 
- Desarrollo de fuentes de información alternativas a la escuela, como son la televisión y el Internet que modifican el rol que tradicionalmente la sociedad le ha asignado a educadores y educadoras, creando inseguridad y desconcierto en la función docente.

- Escasez de recursos materiales y deficientes condiciones de trabajo, sobre todo, debido al debilitamiento del rol del Estado en cuanto al financiamiento de la educación, pero a la vez producto de los procesos de expansión de la cobertura educativa.

- Cambios en la relación profesorado - estudiantado, al aumentarse una serie de conflictos disciplinarios que victimizan tanto a docentes como estudiantado.

- Fragmentación del trabajo del profesorado, al tener que asumir simultáneamente labores administrativas, pedagógicas y extracurriculares.

\section{Desarrollo vocacional y profesional de educadores y educadoras}

Todo ser humano, para alcanzar su equilibrio emocional, físico, psíquico y social debe, en cada etapa de la vida, enfrentar y realizar elecciones, según el período de desarrollo en que se encuentra. En el caso del profesorado, se pueden citar la elección de carrera y la adaptación positiva en el campo laboral. Para el logro de esos objetivos, se debe realizar un proceso continuo de descubrimiento de habilidades, intereses y aptitudes que le permita el conocimiento propio y adaptarse positivamente a su campo laboral.

Un aspecto del desarrollo humano que es de primordial importancia para la Orientación, es el desarrollo vocacional. Este se entiende como el proceso que se va dando a lo largo de la vida, de acuerdo con el mismo desarrollo humano, y que busca satisfacer las necesidades relacionadas con la elección de estilos de vida, acorde con el proyecto de vida (Pereira, 1998).

Al respecto, Súper, citado por Osipow (1990), hace referencia al concepto de madurez vocacional, el cual se define como la capacidad individual, tanto cognoscitiva como afectiva, que permite enfrentar con éxito las tareas correspondientes a cada etapa del desarrollo vocacional según las expectativas de la sociedad. Otro elemento del desarrollo vocacional y profesional es el logro de la autoafirmación profesional, entendida como la capacidad desarrollada mediante la formación y el ejercicio laboral, para actuar con autonomía y madurez por medio de la toma de decisiones, en busca de soluciones pertinentes a los problemas que enfrenta el trabajador o la trabajadora profesional (Mata, 2003).

Fomentar estas condiciones personales a lo largo de la formación inicial del profesorado, puede favorecer el enfrentamiento constructivo y creativo de la tarea docente, 
con miras no solo al mejoramiento de la calidad de la educación, sino también como medio para el logro de la satisfacción laboral.

\section{La importancia del papel de la Orientación}

Ante una sociedad en continuo cambio y trasformación donde el mercado de trabajo se ha diversificado, el papel de la Orientación es muy importante en la educación superior.

La Orientación ayuda en el conocimiento del sí mismo, el conocimiento de la realidad y las oportunidades profesionales para lograr en un futuro la inserción laboral. Por lo tanto es tarea de la orientación favorecer el ajuste persona-formación-empleo. Para efectos de este estudio la orientación en la universidad es entendida como lo define Toscano (2002), como un proceso continuo de intervención, conscientemente programado en respuesta a unas necesidades evaluadas previamente en el contexto social y universitario.

Se entiende por necesidades de orientación los retos y soluciones al crecimiento y desarrollo del estudiantado universitario, a la prevención de conductas destructivas que afectan su salud mental, y al balance entre estas dos áreas de la vida estudiantil. Es decir son las estrategias para solventar las carencias del desarrollo e incentivar las fortalezas que permitan bienestar integral (Archer y Cooper, 1998). Sobre la base de la definición de estrategia educativa que propone Blázquez (1997), se puede definir una estrategia de orientación como un sistema de relaciones sobre el que se basa el proceso de orientación, en el que cada profesional es responsable de crearlas, suscitarlas, avivarlas y dirigirlas mediante la planificación y dirección de acciones conscientes e intencionadas, aplicando un determinado enfoque de orientación, para conseguir los objetivos establecidos que pretenden facilitar el desarrollo de las personas.

Pereira (1998) explica dos grandes estrategias para incorporar la Orientación al currículo o al centro educativo. La primera se denomina adición, en cuyo caso la orientación es una materia más del plan de estudios, o una intervención particular por parte de sus profesionales. La segunda es por infusión, la cual consiste en influir en el currículo como un todo, mediante la incorporación de este enfoque en todas las materias; la importancia de esta modalidad radica en el diseño de programas coherentes, secuenciales y con cobertura para todos los elementos del currículo. En este caso, el profesorado universitario es el responsable de realizar la tarea orientadora.

Como agentes facilitadores de la Orientación en la Universidad de Costa Rica se destaca la Oficina de Orientación a la que le corresponde dirigir, agrupar, coordinar y evaluar los servicios y proyectos de orientación al estudiantado. La Oficina de Orientación tiene 
como propósito promover un proceso de orientación integral para ayudar al estudiante en la construcción de respuestas a necesidades que surgen durante su formación. Para lograr estos propósitos se divide en tres tipos de Unidades Operativas: Centro De Orientación Vocacional (C.O.V.O.), Centros De Asesoría Estudiantil (C.A.S.E.), Centros De Asesoría Y Servicios Al Estudiante Con Discapacidad (C.A.S.E.D.) (Vicerrectoría de Vida Estudiantil, 2001).

Otro agente facilitador del proceso de orientación en la Universidad de Costa Rica, es el profesor universitario, quien realiza una función de asesoría y acompañamiento al estudiantado, desde que éste inicia los estudios hasta que finaliza la formación profesional, de acuerdo con los reglamentos vigentes en la institución (UCR, 2001).

\section{DESCRIPCIÓN METODOLÓGICA}

Para la identificación de las necesidades de orientación de un grupo de estudiantes de la Escuela de Formación Docente de la Universidad de Costa Rica, se realizó una investigación descriptiva enmarcada en la investigación social cualitativa, por ser coherente con los objetivos de la presente investigación. Este grupo de participantes en conjunto con el equipo de investigación, elaboró sus propios significados sobre tales necesidades, asociadas a su formación inicial, y con miras al enfrentamiento constructivo de los retos y demandas del sistema educativo costarricense. Se utilizó como técnica de recolección de la información los grupos de discusión, y el análisis de contingencias como técnica de análisis de la información.

La técnica de grupos de discusión, según Canales y Peinado (1994 ) es un tipo de entrevista grupal que trabaja con el habla. Equivale a una conversación guiada con personas que poseen ciertas características y que ofrecen datos, lo que dicen es motivo de reflexión y análisis. En ella se concentra la diversidad de actitudes, experiencias y creencias del grupo de participantes del estudio. Se utilizó la técnica del muestreo teórico que indica que el número de "casos" estudiados carece relativamente de importancia, sino más bien el potencial de cada "caso" como medio para la comprensión teórica del problema en estudio (Taylor y Bogdan, 1996).

El objetivo general de esta técnica de recolección de información consistió en detectar opiniones y argumentos, ajustados a los resultados de las observaciones y vivencias del grupo de participantes para dar respuesta al problema de investigación. En síntesis el trabajo buscó tener información que facilitase la atención particular de las necesidades de orientación de la población estudiantil, de las carreras de Formación Docente de la 
Universidad de Costa Rica, en su periodo de formación inicial, por parte de la Oficina de Orientación y del profesorado universitario que atiende esta población.

Para la interpretación de los resultados se utilizó la técnica de análisis de contingencias (Navarro y Díaz, 1994), la cual permitió la elaboración de unidades de registro como unidades de significación de los contenidos expuestos por quienes participaron en el estudio, y de unidades de contexto como grandes categorías para la relativización y comprensión teórica y contextual de dichos contenidos.

Por último se realizó un proceso de triangulación a partir de la información obtenida en una entrevista grupal que se efectuó con funcionarios y funcionarias del Centro de Asesoría Estudiantil de Ciencias Sociales, con el fin de contar con un tercer criterio sobre la información recolectada y a su vez, completar el análisis de la información y alcanzar la cristalización de los resultados de la investigación.

\section{RESULTADOS}

El primer paso seguido, de acuerdo con la técnica para el análisis de la información, fue la identificación de los temas recurrentes en las participaciones del grupo investigado, lo que dio origen a las siguientes unidades de registro:

1. Apreciación personal sobre la utilidad de lo enseñado y aprendido en la universidad.

2. Reconocimiento de la desarticulación entre teoría y práctica.

3. Apreciación personal sobre la relación profesorado - estudiantado durante la formación inicial.

4. Confrontación entre las expectativas elaboradas en la formación universitaria y la realidad laboral.

5. Compromiso personal en la formación docente.

6. Valoración personal sobre la pertinencia del plan de estudios de formación inicial.

7. Desconcierto ante los diferentes conflictos que se presentan en el ambiente laboral los cuales causan inseguridad.

8. Conciencia sobre la realidad de que la formación inicial es solo el primer paso de un largo camino profesional.

9. Problemas que el grupo profesional en docencia enfrenta, los cuales se encuentran asociados a la iniciación laboral:

a. Sentimiento de impotencia al tratar de ajustar las directrices del MEP con las necesidades del estudiantado a cargo. 
b. Dificultad de manejar las diferencias individuales ante una gran cantidad de estudiantes en el aula.

c. Dificultad para atender adecuadamente a las personas con Necesidades Educativas Especiales.

d. Falta de infraestructura y el equipo adecuado en las instituciones educativas.

e. Poco interés del estudiantado en la materia impartida por lo cual presentan un bajo rendimiento académico.

f. Dificultades para establecer una adecuada relación profesorado-estudiantado, en el ámbito laboral.

g. Falta de conocimiento para enfrentar las necesidades emocionales de la población estudiantil, generadas por las situaciones familiares y socioeconómicas que enfrentan.

h. Dificultad para incorporar metodologías más activas en el trabajo de aula para responder a las nuevas tecnologías.

10. Satisfacción por la ayuda recibida de compañeros y compañeras de trabajo con más experiencia laboral.

11. Mayor concienciación de las razones por los cuales se estudió educación, para automotivarse y asumir de forma positiva la función docente.

12. Compromiso personal con la formación integral del estudiantado poniendo énfasis en los valores.

13. Preocupación por la falta de valorización social de la función docente.

14. Satisfacción por la ayuda que se recibe de los compañeros y compañeras de la universidad en la formación inicial.

Una vez identificadas las anteriores unidades de registro fue preciso crear las unidades de contexto, las cuales se definieron desde el problema de investigación y fundamentadas en los referentes teóricos del estudio. Es decir, al interpretar el contenido de las anteriores unidades de registro, fue posible construir tres grandes tipos de necesidades de orientación de la población estudiada, a saber:

1. Necesidad del desarrollo de fortalezas para la autoafirmación profesional. La autoafirmación profesional es comprendida como la capacidad desarrollada mediante la formación para el ejercicio laboral, para actuar con autonomía y madurez por medio de la toma de decisiones, en busca de soluciones pertinentes a los problemas que enfrenta el trabajador y trabajadora profesional (Mata, 2004). Dentro de esta unidad de contexto se proponen como mecanismos para lograr la autoafirmación el 
conocimiento del ambiente laboral, la identificación con la función docente y el compromiso con la formación continua. Esta unidad de contexto fue conformada por las siguientes unidades de registro: los problemas asociados a la iniciación laboral, la falta de valorización social actual de la función docente, la conciencia sobre la realidad que la formación inicial es solo el primer paso de un largo camino profesional, confrontación entre las expectativas elaboradas en la formación universitaria y la realidad laboral; el compromiso personal con la formación integral del estudiantado, poniendo énfasis en los valores.

2. Necesidad de fortalecer habilidades de vida. Las habilidades de vida son definidas como la utilización de comportamientos apropiados para la resolución de problemas relacionados con asuntos personales, familiares, de tiempo libre, de la comunidad y del trabajo (Bisquerra, 2000). Para efectos de la investigación, se toma como base la habilidades de vida que enuncia este autor, clasificándolas en tres categorías:

a. Las habilidades cognitivas que engloban ocho unidades de registro: los problemas que el profesorado enfrenta asociados con la iniciación laboral; apreciación personal sobre la utilidad de lo enseñado y aprendido en la universidad; valoración personal sobre la pertinencia del plan de estudios de formación inicial; la conciencia sobre la realidad de que la formación inicial es solo el primer paso de un largo camino profesional; confrontación entre las expectativas elaboradas en la formación universitaria y la realidad laboral; el reconocimiento de la desarticulación entre la teoría y la práctica; una mayor concienciación de las razones por las cuales se estudió educación y el compromiso personal en la formación docente.

b. Las habilidades afectivas que incluyen ocho unidades de registro: los problemas que el profesorado enfrenta asociados a la iniciación laboral; la preocupación por la falta de valorización social de la función docente; el desconcierto ante los diferentes conflictos que se presentan en el ambiente laboral los cuales causan inseguridad; la apreciación personal sobre la relación profesorado-estudiantado durante la formación inicial; la confrontación entre las expectativas elaboradas en la formación universitaria y la realidad laboral; el compromiso personal en la formación integral del estudiantado poniendo énfasis en los valores; la satisfacción por la ayuda recibida de los compañeros y las compañeras de la universidad en la 
formación inicial; la satisfacción por la ayuda recibida de compañeros y compañeras de trabajo con más experiencia laboral.

c. Las habilidades organizacionales que incluyen tres unidades de registro: los problemas que enfrenta el profesorado asociados a la iniciación laboral; el desconcierto ante los diferentes conflictos que se presentan en el ambiente laboral, los cuales causan inseguridad y la confrontación entre las expectativas elaboradas en la formación universitaria y la realidad laboral.

3. Necesidad de fortalecer la madurez vocacional. La madurez vocacional puede definirse como la capacidad individual, tanto cognoscitiva como afectiva, que permite enfrentar con éxito las tareas correspondientes a cada etapa del desarrollo vocacional según las expectativas de la sociedad. Aunque el estudiantado considere clara su decisión, los eventos posteriores podrían suscitar cambios tardíos e impredecibles. Por lo tanto, debe confirmar su identidad profesional con una visión acorde con sus intereses y metas de vida (Super citado por Osipow, 1990). Esta última unidad de contexto incluye los mecanismos de profundizar el conocimiento de sí mismo, examinar los motivos por los cuales se estudió educación y la capacidad para tomar decisiones efectivas.

\section{UNA PROPUESTA DE ORIENTACIÓN}

El objetivo principal de la presente investigación consistió en detectar las necesidades de orientación en la formación inicial de un grupo de estudiantes de formación docente de la Universidad de Costa Rica, ante los cambios y desafíos del sistema educativo costarricense; y con ello favorecer al deseado ajuste persona-formación- empleo.

En este sentido, la función orientadora facilita al estudiantado el desarrollo adecuado del conocimiento de sí mismo y la comprensión del contexto (condiciones de trabajo y la realidad sociocultural del entorno) para poder integrar esta síntesis en la planificación de proyectos de vida personal y profesional, de forma autónoma y responsable con su entorno social.

En consecuencia, la orientación profesional debe dar respuesta a los siguientes retos: contribuir a la relación de intercambios entre el sistema educativo y el mundo del trabajo, para asegurar una adecuada transición de la formación al empleo; fortalecer la madurez personal que le permita a la población estudiantil tomar decisiones autónomas y acordes con sus intereses personales y las demandas sociales; incrementar la calidad del sistema educativo y formativo de las universidades, entre otras. 
De esta manera, la orientación le permite a la formación universitaria una visión más adecuada del mundo del trabajo, de las demandas sociales hacia las profesiones, de los aprendizajes más significativos del estudiantado. Así, la orientación da valiosos aportes a la formación inicial del grupo de estudiantes de formación docente.

La orientación debe basarse en necesidades evaluadas previamente en el contexto social y universitario. Este proceso se realizó en la presente investigación en donde se caracterizaron las siguientes necesidades de orientación en la población estudiada: autoafirmación profesional, habilidades de vida y madurez vocacional.

Las necesidades detectadas no solamente se limitan al ámbito universitario, sino también están circunscritas a una mejor adaptación al campo laboral y a enfrentar las demandas sociales relacionadas con la función docente.

Se considera que las estrategias que mayormente responderían a las necesidades mostradas por los sujetos de estudio, deben dirigirse en las dos líneas de acción antes mencionadas: la adición y la infusión. La adición implica que la orientación sea un servicio más dentro de la formación de la población estudiantil universitaria. Se dirige a realizar tareas de orientación específicas y especializadas del departamento orientación. Por su parte la infusión consiste en influir en el currículum como un todo, diseñando programas coherentes y secuenciales; por ejemplo, acciones dirigidas a complementar la enseñanza de las asignaturas; el compromiso de la orientación como agente de cambio en las instituciones educativas; el asesoramiento al profesorado y equipos educativos, entre otros.

Para dar respuesta a las necesidades detectadas en el estudio, se definirán estrategias de orientación que puedan ser desarrolladas por dos agentes muy importantes del ambiente universitario: el profesorado y la Oficina de Orientación.

\section{Necesidad de autoafirmación profesional}

La autoafirmación profesional es comprendida como la capacidad desarrollada, mediante la formación para el ejercicio laboral, para actuar con autonomía y madurez por medio de la toma de decisiones, en busca de apoyos para dar soluciones pertinentes a los problemas que enfrenta el trabajador y la trabajadora profesional. Se puede contribuir a la autoafirmación profesional del grupo de estudiantes de formación docente, cuando se le facilita el conocimiento del ambiente laboral, la identificación con la función docente y la posibilidad de adquirir un compromiso con la formación continua.

Desde la función orientadora del profesorado, para favorecer la autoafirmación profesional, se considera importante propiciar contactos con la realidad laboral, desde los 
primeros cursos de la formación inicial, y así contribuir a un aprendizaje teórico-práctico, adaptado a las exigencias actuales de la función docente; crear espacios de realimentación donde la población estudiantil comparta sus expectativas y dudas sobre el ambiente laboral y su función docente; concienciar al estudiante sobre las diferentes funciones que realizará en su quehacer laboral (formar en valores, atender las necesidades emocionales del estudiantado, entre otras). Además de la función exclusivamente instructiva, incentiva a la población estudiantil de formación docente a valorar la importancia de su función docente, como un agente de cambio social y de formador de las futuras generaciones; motivar al estudiantado a la toma de conciencia de que la preparación universitaria, es solo el primer paso de una formación a lo largo de su vida como profesional; y crea un espacio de diálogo donde se exprese la desvalorización social de la labor docente y así rescatar los sentimientos de insatisfacción y decepción que manifestaron el grupo de estudiantes participantes en la investigación.

Por su parte la Oficina de Orientación puede implementar instrumentos o proyectos que permitan al estudiantado de formación docente, bajar la ansiedad y el temor creados por la transición del ambiente educativo al mundo del trabajo, de manera que la población estudiantil encuentre un apoyo en esta nueva etapa, ampliando su conocimiento del medio laboral y reafirmando su elección de ser docente.

Además, una labor que pueden desarrollar los centros de asesoría estudiantil es facilitar asesoría a las asociaciones estudiantiles para reforzar la identidad profesional, respaldado en sus funciones de coordinar con unidades académicas y organizaciones estudiantiles para la ejecución de proyectos y servicios; proponer, analizar y desarrollar y apoyar proyectos y servicios que promueven la incorporación, el desarrollo académico, personal y convivencia del estudiantado; y brindar apoyo técnico profesional a las unidades académicas para contribuir en el desarrollo de los proyectos.

Como aporte específico del proceso de triangulación realizado para la validación de la información, se destacan cuatro ideas fundamentales que reafirman los resultados de la investigación: un adecuado conocimiento del mundo laboral; procesos de búsqueda de empleo e inducción al mundo del trabajo; la responsabilidad del profesorado universitario como formador más allá de la transmisión de conocimientos y la responsabilidad del estudiantado con su formación; desvalorización social de la educación; y la formación universitaria que debe responder a las demandas sociales. 


\section{Necesidad de fortalecer las habilidades de vida}

Las habilidades de vida son definidas como la utilización de comportamientos apropiados para la resolución de problemas relacionados con asuntos personales, familiares, de tiempo libre, de la comunidad y del trabajo. Para la promoción de habilidades cognitivas corresponde al profesorado universitario fortalecer en el grupo de estudiantes de formación docente la habilidad para encontrar información y recursos; pensar y resolver problemas constructivamente; identificar su potencial creativo y desarrollarlo; fortalecer la capacidad de análisis, síntesis y evaluación; fomentar el pensamiento crítico y flexible; desarrollar la capacidad investigativa- reflexiva y estimular la iniciativa.

Estas habilidades le permitirán a la población estudiantil de formación docente una mejor transición de la formación inicial, al mundo de trabajo. Específicamente podrá asumir un papel protagónico y activo en su aprendizaje; integrar sus características personales a las demandas académicas en la formación inicial; disminuir los efectos propios del choque con la realidad; enfrentar de forma adecuada las diferentes situaciones que surgen en el trabajo de aula y adecuar lo aprendido en la formación inicial a la practica laboral.

Por su parte, el grupo de profesionales en orientación puede implementar espacios que le permitan al estudiantado estimular su creatividad, un pensamiento crítico y flexible y así promover en esta población un papel activo y responsable con su aprendizaje.

También el personal docente universitario, mediante su función orientadora, puede fortalecer habilidades afectivas en el estudiantado de formación docente, entre las cuales se pueden mencionar: desarrollar la capacidad para dar y recibir ayuda en su labor docente; manejar conflictos de forma adecuada; aprender a comunicarse de forma asertiva; fortalecer habilidades para tolerar la frustración; influir en la gente y en los sistemas e inspirar confianza en los demás.

Esto requiere que el profesorado universitario ofrezca un clima de confianza dentro del aula, lo cual le permitirá al estudiantado expresar sus expectativas, sentimientos, pensamientos, temores y dificultades, en relación con su futura labor docente.

Otro aspecto importante que el docente debe propiciar dentro del aula, es la realimentación entre el grupo de compañeros y compañeras de clase (compañero mentor), que puedan ser un apoyo emocional en la resolución de conflictos y en la búsqueda de soluciones ante los problemas propios de la formación, así como, la colaboración en los procesos propios del proceso educativo.

Al fortalecer estas habilidades, se desarrollan en la población estudiantil de formación docente, más herramientas para poder enfrentar de forma adecuada todas las situaciones 
que se presenten en el mundo laboral (conflictos con la autoridad, entre compañeros y compañeras, con los padres y madres de familia, entre otros), y así disminuir el choque con la realidad.

Respecto al aporte de las instancias que ofrecen servicios de orientación, éstas se constituyen en el ente más capacitado para desarrollar habilidades afectivas, tales como: aprender a manejar las emociones negativas; enfrentar en forma positiva el estrés; desarrollar la tolerancia a la frustración, capacidad para dar y recibir afecto, manejar conflictos en forma adecuada y aprender a expresar sentimientos en forma constructiva.

Todo esto se presenta con el fin de que la población estudiantil de formación docente logre potenciar sus habilidades de vida y sociales (madurez emocional), manifestándose como personas más autorrealizadas y con un equilibrio emocional propio de las exigencias de su función docente. De esta manera, es importante resaltar las siguientes funciones de esta unidad operativa: brindar atención profesional personalizada, acorde con las características de la situación del estudiantado; estudiar aspectos teóricos y técnicos, relativos a las necesidades y los requerimientos del estudiantado que permitan definir estrategias de intervención; y realizar su labor en forma interdisciplinaria, con base en las características de la población que atiende.

Otras habilidades de vida importantes son las habilidades organizacionales, las cuales le permiten al estudiantado de formación docente estar mejor preparado para cumplir las tareas y funciones que se le asignen en el sistema educativo. Entre ellas, se presentan conocer los procedimientos y la información pertinente para insertarse en el mundo del trabajo; retomar los recursos personales desarrollados en la formación inicial y adaptarlos al campo laboral; distribuir el tiempo con efectividad; además de conocer y aprender a realizar las tareas administrativas inherentes a la función docente.

El profesorado universitario puede fomentar estas habilidades al integrar en su quehacer educativo, información sobre la realidad del sistema educativo costarricense, y así el estudiantado de formación docente logrará adaptarse mejor a las situaciones laborales y los requerimientos que enfrentará en su desempeño laboral, tales como: el proceso de reclutamiento en el Ministerio de Educación Pública; la integración a las asociaciones magisteriales; la ejecución de tareas administrativas y el conocimiento de las leyes que respaldan su desempeño laboral.

Es oportuno reforzar, por parte de las instancias responsables de la orientación vocacional del estudiantado, la importancia que tiene en el ajuste persona-formación-empleo, al brindar información pertinente y de acuerdo con las inquietudes, respecto a los 
procedimientos de inserción al mundo del trabajo, específicamente en el caso de la población estudiantil de formación docente, su mayor empleador el Ministerio de Educación Pública.

Para lograr el cumplimiento de estos objetivos, se puede brindar apoyo mediante talleres de preparación para la búsqueda de empleo; ofrecer servicios de intermediación de empleo mediante un sistema informático; asesoría en herramientas para la búsqueda de empleo, la cual comprende la elaboración de un currículo y la preparación para una entrevista de trabajo, información sobre los colegios profesionales, y los ambientes ocupacionales entre otras.

Como aporte específico del proceso de triangulación, se destacan tres ideas fundamentales que validan y reafirman los resultados de la investigación; a saber, un adecuado conocimiento del mundo laboral; procesos de búsqueda de empleo e inducción al mundo del trabajo; desarrollo de habilidades de vida como por ejemplo la creatividad, la innovación, la iniciativa; habilidades afectivas para que el estudiantado pueda aplicar en la práctica, lo aprendido en la universidad y así disminuya el choque con la realidad y desarticulación entre la teoría y la práctica.

\section{Necesidad de fortalecer la madurez vocacional}

Anteriormente se había definido la madurez vocacional como la capacidad individual, tanto cognoscitiva como afectiva, que permite enfrentar con éxito las tareas correspondientes a cada etapa del desarrollo vocacional, según las expectativas de la sociedad, en donde la persona pueda confirmar su identidad profesional, con una visión acorde con sus intereses y metas de vida. La madurez vocacional se alcanza en esta etapa de desarrollo, cuando el estudiantado profundiza en el conocimiento de sí mismo (intereses, valores, creencias y limitaciones.), del entorno laboral y del medio educativo; examina los motivos por los cuales estudia educación; desarrolla la capacidad para tomar decisiones efectivas y acordes con el proyecto de vida personal.

Para fortalecer la madurez vocacional, el profesorado universitario puede brindar espacios donde el estudiante pueda reflexionar y analizar si sus características personales se ajustan a las demandas del medio laboral y si este cumple con las expectativas de su proyecto de vida profesional. Igualmente, el profesorado durante todo el proceso de formación inicial, puede reforzar el perfil del y la docente, brindándole al estudiantado el conocimiento de valores, actitudes y tareas propios de la tarea docente, enfatizando en la importancia que tiene la profesión, como agente de cambio social. 
Otro aporte significativo relacionado con la madurez vocacional es introducir, desde el inicio, contactos con el campo laboral, de manera que la población estudiantil de formación docente experimente un acercamiento a la diversidad cultural que encontrarán dentro de una institución educativa, distinguiendo diferentes ámbitos (tanto socioeconómicos como culturales). Esto le permitirá al estudiantado crear expectativas reales con respecto al mundo del trabajo y así disminuir los efectos del choque con la realidad.

Todo esto contribuye a enriquecer la visión que tenga la población estudiantil sobre la realidad laboral, permitiéndole encontrar coherencia en su proyecto de vida, así como las fortalezas que le permitan convertirse en ese protagonista generador de cambio social.

Las instancias que brindan servicios de orientación contribuyen en gran parte a responder a las exigencias de la madurez vocacional de la población de formación docente, al ser un apoyo complementario del proceso de discernimiento vocacional, de manera que logre facilitar funciones orientadoras relacionadas con el conocimiento propio de cada estudiante; el acercamiento del entorno laboral; la exploración de los motivos por los cuales estudia educación; y la ayuda al proceso para tomar decisiones efectivas y acordes con el proyecto de vida personal.

De esta manera se puede fortalecer la madurez vocacional mediante la edición de materiales informativos, fichas profesiográficas, además de la realización de ferias vocacionales para los candidatos y candidatas a ingresar a la universidad y la atención de estudiantes colegiales en la medida de lo posible; la creación de sistemas de exploración vocacional computarizados con base de datos de carreras y ocupaciones; información impresa y audiovisual; atención al estudiantado de primer ingreso o de segundo año, por medio de la visita al cien por ciento de los grupos de los primeros cursos universitarios con la intención de que cada estudiante revise su proyecto vocacional. Asimismo se deben coordinar visitas a cursos de carreras a solicitud de las unidades académicas que lo requieran, sin obviar la atención individual o grupal a los estudiantes que desean trasladarse de carrera y la disposición de información de carreras y ocupaciones. Del mismo modo, es importante resaltar la atención de las necesidades del estudiantado que ha superado el segundo año de carrera en sus dudas vocacionales.

Como aporte específico del proceso de triangulación, se destacan seis ideas fundamentales que validan y reafirman los resultados de la investigación: un adecuado conocimiento del mundo laboral; procesos de búsqueda de empleo e inducción al mundo del trabajo; responsabilidad del profesorado universitario como formador más allá de la transmisión de conocimientos y la responsabilidad del estudiante con su formación; 
identificación con un perfil profesional; crear espacios donde el estudiantado pueda evaluar su decisión vocacional para contribuir a su madurez vocacional; la tecnología como un nuevo reto para el profesorado; y la formación universitaria que debe responder a las demandas sociales.

\section{CONCLUSIONES}

A continuación se destacan las principales conclusiones del estudio:

1. Mediante la presente investigación se caracterizaron las siguientes necesidades de orientación en la población estudiantil estudiada: fortalecimiento de la autoafirmación profesional, el desarrollo de habilidades de vida y la consolidación de la madurez vocacional esperada en esta fase del desarrollo vocacional.

2. Se puede contribuir al fortalecimiento de la autoafirmación profesional del estudiantado participante en este estudio, facilitando el conocimiento del ambiente laboral, estimulando la identificación con la función docente y favoreciendo la adquisición de un compromiso con la propia formación continua.

3. Se contribuye el fortalecimiento de las habilidades de vida de la población estudiantil participante en el estudio al facilitar la utilización de comportamientos apropiados para la resolución de situaciones relacionadas con asuntos personales, familiares, de tiempo libre, de la comunidad y del trabajo.

4. El desarrollo de la madurez vocacional propia de la etapa del desarrollo en que se encuentra esta población estudiantil se estimula al profundizarse el análisis de sí mismo, del entorno laboral y del medio educativo. Además cuando se examinan los motivos por los cuales se ha elegido la carrera de educación, y además se desarrolla la capacidad para tomar decisiones efectivas y acordes con el proyecto de vida personal.

5. Un aporte significativo que retoma la presente investigación es utilizar la infusión y la adición como estrategias que facilitan la labor orientadora. Para complementar dicha labor, se requiere de la función orientadora de los agentes muy importantes en el ambiente universitario: el personal docente y los profesionales y las profesionales de la Oficina de Orientación.

6. La propuesta de Orientación que se propone como resultado de esta investigación, a la vez que estimula un mejor aprovechamiento del proceso de formación universitaria, permite a los futuros y las futuras profesionales desarrollar recursos para dar respuestas constructivas a las exigencias actuales del campo laboral, y cumplir con los objetivos de su proyecto de vida personal y profesional. 
7. La formación incial debe contribuir a forjar en los estudiantes de formación docente, un conjunto de destrezas y actitudes que les permitan alterar, recrear y reconvertir continuamente los contextos culturas, sociales y educativos en los que les corresponda ejercer su labor profesional, reforzando el papel del educador como agente de cambio social. También debe relacionarse directamente con los resultados de la investigación

\section{REFERENCIAS}

Apodaca, P.; Lobato, C. (1997). Calidad en la universidad: orientación y evaluación. Barcelona: Editorial Alertes.

Archer, J.; Cooper, S. (1998). Counseling and Mental Health Services on Campus. San Francisco, USA: Jossey-Bass Publisher.

Bisquerra, R. (2000). Educación emocional y bienestar. Barcelona: Praxis.

Blázquez, F y otros. (1997). Formación psicopedagógica del profesorado de secundaria. Extremadura, España: Instituto de Ciencias de la Educación.

Canales, M.; Peinado, A. (1994). Grupos de discusión. En Métodos y técnicas cualitativas de investigación en Ciencias Sociales. Juan Manuel Delgado y Juan Gutiérrez, coordinadores. Madrid: Editorial Síntesis S.A.

Estado de la Nación. (2002). Octavo informe del Estado de la Nación en Desarrollo Humano Sostenible. San José: Proyecto Estado de la Nación.

Esteve, J.M.; Franco, S.; Vera, J. (1995). Los profesores ante el cambio social. Barcelona: Editorial Anthropos.

Imbernón, F. (1994). La formación y el desarrollo profesional del profesorado. Barcelona: Editorial Graó.

Marcelo García, C. (1994). Formación del profesorado para el cambio educativo. Barcelona: PPU, S.A.

Mata Segreda, A. (2003). La Orientación y el mejoramiento de la capacidad para el desempeño laboral del trabajador. Actualidades en Orientación. 7(1): 5-8. Asociación Costarricense de Profesionales en Orientación.

Navarro, P.; Díaz, C. (1994). Análisis de contenido. Métodos y técnicas cualitativas de investigación en Ciencias Sociales. Juan Manuel Delgado y Juan Gutiérrez, coordinadores. Madrid: Editorial Síntesis.

Osipow, S. (1990). Teorías sobre la elección de carreras. México: Trillas.

Pereira, T. (1998). Orientación educativa. San José: Editorial de la Universidad Estatal a Distancia. 
Taylor, S.J.; Bogdan, R. (1996). Introducción a los métodos cualitativos de investigación. Barcelona: Paidos.

Toscano Cruz, M. (2002). Necesidad de la Orientación en la Universidad. Universidad de Huelva, España. Recuperado en el mes de setiembre de octubre de 2002, de http://www2.uhu.es/agora/digital/numeros02/02-artículos/monográfico/mariola.htm.

Universidad de Costa Rica. (2001). Reglamento de régimen académico estudiantil. En Compendio de normativa universitaria. Universidad de Costa Rica: Oficina Jurídica.

Vicerrectoría de Vida Estudiantil. (2001). Descripción de la Estructura y Funcionamiento de la Oficina de Orientación. San José: Universidad de Costa Rica. 